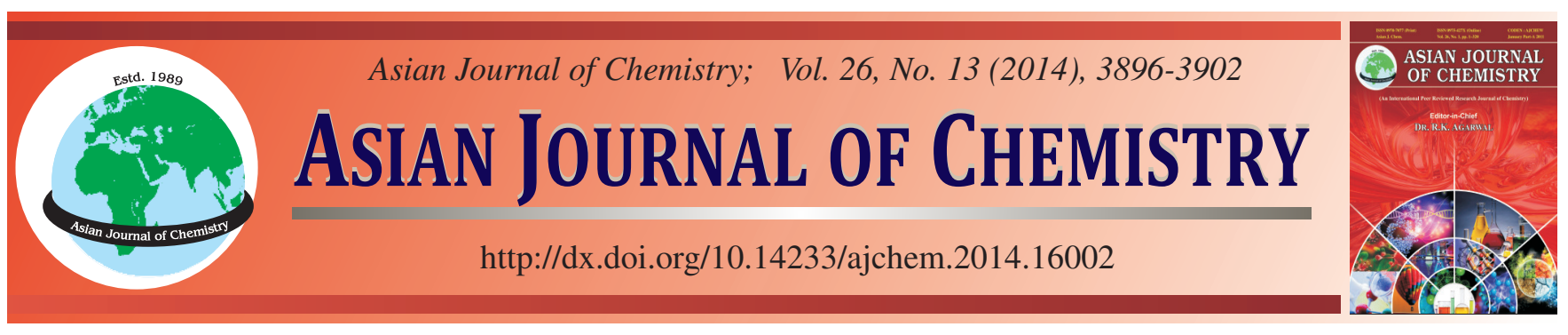

\title{
Synthesis of Some Novel Pyrazolopyridooxazine, Pyrazoloquinolizines, Pyrazoloindolizine and Pyrazolopyranopyrimidinone Derivatives
}

Hissah H. Al-Tilasi ${ }^{*}$ and Fatma E.M. El-BaiH

Women Students-Medical Studies \& Sciences Sections, Chemistry Department, College of Science, King Saud University, P.O. Box 22452, Riyadh 11495, Saudi Arabia

*Corresponding author: Fax: +966 1 4772245; Tel: +966 503456589; E-mail: haltalassi@ksu.edu.sa

Received: 4 July 2013;

Accepted: 16 October 2013;

Published online: 23 June 2014;

AJC-15369

Different pyrazolone derivatives were prepared as starting materials for the synthesis of pyrazolopyridooxazine, pyrazoloquinolizines,
pyrazoloindolizine, 1,4-oxazinopyrazolines and pyrazolopyranopyrimidinone derivatives via reactions with different reagents applying
the one pot multicomponent reaction using microwave and ultrasound irradiation in some cases.

Keywords: Chloropyrazolecarbaldehyde, Pyrazolopyridooxazine, Pyrazoloquinolizines, Pyrazoloindolizine, Pyrazolopyranopyrimidinone.

ᄂ - - - - - - - - - - - - - - - - - - - - - - - - -

\section{INTRODUCTION}

Several heterocyclic compounds containing pyrazole ring are very useful as intermediates for pharmaceuticals ${ }^{1-4}$ and have shown several biological activities such as antiinflammatory agents $^{5-7}$, antibacterial ${ }^{8-12}$, antifungal ${ }^{9}$, antiviral ${ }^{13,14}$, antitumor ${ }^{15-19}$ and insecticidal ${ }^{20,21}$. Numerous pyrazole derivatives are also used in dye industry ${ }^{22}$. In our continued interest in the development of highly expedient methods for the synthesis of diverse heterocyclic compounds of biological significance, we report herein the synthesis of some novel pyrazole derivatives which are pyrazolinoquinolizines, 1,4-oxazinopyrazolines, 6-amino-1,4dihydro-pyrano[2,3-c]pyrazole-5-carbonitrile and 4,6dihydropyrazolo[4',3':5,6]pyrano[2,3-d]pyrimidin-5(1H)-one. Moreover, in some cases we used a one pot multicomponents reaction using microwave and ultrasound irradiation.

\section{EXPERIMENTAL}

Melting points were determined using an Electrothermal IA9000 series digital capillary melting point apparatus and are uncorrected. IR spectra were obtained as $\mathrm{KBr}$ discs on a 1000-Perkin Elmer FT-IR spectrophotometer. ${ }^{1} \mathrm{H}$ NMR and ${ }^{13} \mathrm{C}$ NMR spectra were recorded on a JEOL ECP-300 NMR in $\mathrm{CDCl}_{3}$ (or DMSO- $d_{6}$ ) using TMS as an internal standard. Chemical shifts are given in ppm on the $\delta$ scale and coupling constants $(J)$ are given in Hz. Electron impact (EI) MS spectra were acquired with the aid of a Shimadzu GCMSQP5050A spectrometer, equipped with a $30 \mathrm{~m} \times 0.25 \mathrm{~mm}$ DB- 1 glass column, operating with an ionization energy of $70 \mathrm{eV}$, at the
Chemistry Department, College of Science, King Saud University. Electrospray ionization (ESI) MS spectra were acquired with LC-MSMS Acquity UPLC/Qutlro Premier Xe at research center King Faisal Specialist Hospital. Ultrasonication was performed in a J.P. Selecta ultrasound cleaner with a frequency of $60 \mathrm{~Hz}$ and an output power of, $770 \mathrm{~W}$. Microwave irradiated reactions were carried out on a Galanz microwave oven, operating at $1000 \mathrm{~W}$, generating $60 \mathrm{~Hz}$ frequency.

Synthesis of 1,3-disubstituted $1 \mathrm{H}$-pyrazol-5(4H)-one (2a-f): They were synthesized by reaction of $\beta$-ketoester with appropriate hydrazines according to the literature procedures ${ }^{23}$.

Synthesis of 5-chloro-3-substituted 1-phenyl-1Hpyrazole-4-carbaldehyde (3a-d): They were synthesized from the 5-pyrazolones employing Vilsmeier-Haack chloroformylation $^{24}$.

Synthesis of 3,5-disubstituted-1-phenyl-1H-pyrazole4-carbaldehyde (4a-e): A mixture of 3a-d (10 mmol) and cyclic sec-amines (viz., morpholine, piperidine and pyrrolidine) $(5 \mathrm{mmol})$ in dry ethanol $(20 \mathrm{~mL})$ containing dry triethylamine $(1 \mathrm{~mL})$ was heated under reflux for 17-22 h (monitored by TLC). After completion, ethanol was removed in a rotavapor, the residue was treated with water and was extracted with dichloromethane $(3 \times 20 \mathrm{~mL})$. The combined organic phases were dried over anhydrous sodium sulfate overnight and concentrated in vacuo. The product was then subjected to column chromatography to afford pure $4 \mathbf{a}-\mathbf{e}^{25}$.

3-Methyl-5-morpholin-4-yl-1-phenyl-1H-pyrazole-4carbaldehyde (4a): Fine pale yellow cubes, m.p. 109-111 ${ }^{\circ} \mathrm{C}$; Yield $13 \%$; IR (KBr, $\left.v_{\max }, \mathrm{cm}^{-1}\right): 2851(\mathrm{CHO}), 1656(\mathrm{C}=\mathrm{O})$; 
${ }^{1} \mathrm{H}$ NMR $\left(\mathrm{CDCl}_{3}\right): 2.45\left(3 \mathrm{H}, \mathrm{s}, \mathrm{CH}_{3}\right), 3.13(4 \mathrm{H}, \mathrm{t}, J=4.6$, $\left.\mathrm{CH}_{2}-\mathrm{N}-\mathrm{CH}_{2}\right), 3.67$ (4H, t, $\left.J=4.6, \mathrm{CH}_{2}-\mathrm{O}-\mathrm{CH}_{2}\right), 7.38-7.50(5 \mathrm{H}$, m, Ar-H), $9.95(1 \mathrm{H}, \mathrm{s}, \mathrm{CHO}) ;{ }^{13} \mathrm{C}$ NMR: $12.9\left(\mathrm{CH}_{3}\right), 50.1,66.0$ (morpholinyl group carbons), 111.3, 124.2, 127.7, 128.5, 138.3,151.0, 151.5 ( $s p^{2}$ carbons), $182.5(\mathrm{C}=\mathrm{O}) ; \mathrm{MS}: \mathrm{m} / \mathrm{z}(\%)$ $271\left[\mathrm{M}^{+}\right]$(18) $\left(\mathrm{C}_{15} \mathrm{H}_{17} \mathrm{~N}_{3} \mathrm{O}_{2}\right), 226\left[\mathrm{M}-\mathrm{CH}_{3}-\mathrm{CHO}-\mathrm{H}\right]$ (7), 211 $\left[\mathrm{M}-\mathrm{C}_{3} \mathrm{H}_{6} \mathrm{O}-2 \mathrm{H}\right](28), 143\left[\mathrm{M}-\mathrm{C}_{2} \mathrm{H}_{3} \mathrm{~N}-\mathrm{HCN}\right](36), 91\left[\mathrm{C}_{6} \mathrm{H}_{5} \mathrm{~N}^{+}\right]$ (36), $77\left[\mathrm{C}_{6} \mathrm{H}_{5}^{+}\right](82), 51\left[77-\mathrm{C}_{2} \mathrm{H}_{2}\right]$ (100).

5-Morpholin-4-yl-1,3-diphenyl-1H-pyrazole-4-carbaldehyde (4b): Fine orange cubes, m.p. 147-149 ${ }^{\circ} \mathrm{C}$; Yield 31 $\%$; IR (KBr, $\left.v_{\max }, \mathrm{cm}^{-1}\right): 2738-2858(\mathrm{CHO}), 1657(\mathrm{C}=\mathrm{O}) ;{ }^{1} \mathrm{H}$ NMR $\left(\mathrm{CDCl}_{3}\right): 3.19\left(4 \mathrm{H}, \mathrm{t}, J=4.6, \mathrm{CH}_{2}-\mathrm{N}-\mathrm{CH}_{2}\right), 3.73(4 \mathrm{H}, \mathrm{t}$, $\left.J=4.6, \mathrm{CH}_{2}-\mathrm{O}-\mathrm{CH}_{2}\right), 7.42-7.56\left(6 \mathrm{H}, \mathrm{m}, \mathrm{H}-3^{\prime}, 3^{\prime \prime}, 4^{\prime}, 4^{\prime \prime}, 5^{\prime}, 5^{\prime \prime}\right)$, $7.66\left(4 \mathrm{H}, \mathrm{dd},{ }^{3} J=7.5,{ }^{4} J=2.9, \mathrm{H}-2^{\prime}, 2^{\prime \prime}, 6^{\prime}, 6^{\prime \prime}\right), 9.82(1 \mathrm{H}, \mathrm{s}$, $\mathrm{CHO}) ;{ }^{13} \mathrm{C}$ NMR: 50.0, 66.3 (morpholinyl group carbons), 110.3, 124.8, 128.0, 128.5, 128.7, 131.1, 138.6, 150.5, 155.5 ( $s p^{2}$ carbons), $183.8(\mathrm{C}=\mathrm{O}) ; \mathrm{MS}: m / z(\%) 333\left[\mathrm{M}^{+}\right]$(18) $\left(\mathrm{C}_{20} \mathrm{H}_{19} \mathrm{~N}_{3} \mathrm{O}_{2}\right), 332$ [M-H] (11), 302 [M-CHO-2H] (24), 274 $\left[\mathrm{M}-\mathrm{C}_{3} \mathrm{H}_{6} \mathrm{O}-\mathrm{H}\right](21), 171\left[274-\mathrm{C}_{7} \mathrm{H}_{5} \mathrm{~N}\right](15), 144$ [171- $\left.\mathrm{HCN}\right]$ (20), $104\left[\mathrm{M}-\mathrm{C}_{13} \mathrm{H}_{14} \mathrm{~N}_{2} \mathrm{O}_{2}+\mathrm{H}\right]$ (42), $91\left[\mathrm{C}_{6} \mathrm{H}_{5} \mathrm{~N}^{+}\right]$(30), 77 $\left[\mathrm{C}_{6} \mathrm{H}_{5}^{+}\right]$(100), 51 [77- $\left.\mathrm{C}_{2} \mathrm{H}_{2}\right]$ (49).

1,3-Diphenyl-5-piperidino-1H-pyrazole-4-carbaldehyde (4c): Fine pale beige cubes, m.p. $115-116^{\circ} \mathrm{C}$; Yield $28 \%$; IR $\left(\mathrm{KBr}, v_{\max }, \mathrm{cm}^{-1}\right)$ : 2752-2852 (CHO), $1669(\mathrm{C}=\mathrm{O}) ;{ }^{1} \mathrm{H} \mathrm{NMR}$ $\left(\mathrm{CDCl}_{3}\right): 1.56\left(6 \mathrm{H}\right.$, br. peak, $3 \mathrm{CH}_{2}$ of the piperidino group), $3.14\left(4 \mathrm{H}\right.$, br. peak, $\left.\mathrm{CH}_{2}-\mathrm{N}-\mathrm{CH}_{2}\right), 7.38-7.58(6 \mathrm{H}, \mathrm{m}, \mathrm{H}-$ $\left.3^{\prime}, 3^{\prime \prime}, 4^{\prime}, 4^{\prime \prime}, 5^{\prime}, 5^{\prime \prime}\right), 7.68$ (4H, dd, $\left.{ }^{3} J=7.5,{ }^{4} J=2.9, \mathrm{H}-2^{\prime}, 2^{\prime \prime}, 6^{\prime}, 6 "\right)$, $9.83(1 \mathrm{H}, \mathrm{s}, \mathrm{CHO}) ;{ }^{13} \mathrm{C}$ NMR: 23.2, 25.3, 51.4 (piperidino group carbons), 110.4, 124.7, 127.7, 128.0, 128.3, 128.6, 131.6, 139.1, 152.4, 155.2 ( $s p^{2}$ carbons), $183.8(\mathrm{C}=\mathrm{O})$; MS: $\mathrm{m} / \mathrm{z}(\%) 331\left[\mathrm{M}^{+}\right](82)\left(\mathrm{C}_{21} \mathrm{H}_{21} \mathrm{~N}_{3} \mathrm{O}\right), 330[\mathrm{M}-\mathrm{H}](29), 302$ [MCHO] (13), $262\left[\mathrm{M}-\mathrm{C}_{5} \mathrm{H}_{10}+\mathrm{H}\right]$ (23), $144\left[\mathrm{M}-\mathrm{C}_{5} \mathrm{H}_{10} \mathrm{~N}-\mathrm{C}_{7} \mathrm{H}_{5} \mathrm{~N}\right]$ (36), $91\left[\mathrm{C}_{6} \mathrm{H}_{5} \mathrm{~N}^{+}\right]$(23), $77\left[\mathrm{C}_{6} \mathrm{H}_{5}{ }^{+}\right]$(100), 51 [77- $\left.\mathrm{C}_{2} \mathrm{H}_{2}\right]$ (64).

3-Ethyl-1-phenyl-5-pyrrolidino- $1 \mathrm{H}$-pyrazole-4carbaldehyde (4d): Fine pale beige cubes, m.p. 82-83 ${ }^{\circ} \mathrm{C}$; Yield $45 \%$; IR $\left(\mathrm{KBr}, v_{\max }, \mathrm{cm}^{-1}\right): 2878(\mathrm{CHO}), 1628(\mathrm{C}=\mathrm{O})$; ${ }^{1} \mathrm{H} \mathrm{NMR}\left(\mathrm{CDCl}_{3}\right): 1.22\left(3 \mathrm{H}, \mathrm{t}, J=7.3, \mathrm{CH}_{3}\right), 2.81$ (2H, quint, $\left.J=7.3, \mathrm{CH}_{2}\right), 1.78-1.83\left(4 \mathrm{H}, \mathrm{m}, 2 \mathrm{CH}_{2}\right.$ of the pyrrolidino group), 3.19-3.23 (4H, m, $\left.\mathrm{CH}_{2}-\mathrm{N}-\mathrm{CH}_{2}\right)$, 7.29-7.39 (5H, m, Ar$\mathrm{H}), 9.92(1 \mathrm{H}, \mathrm{s}, \mathrm{CHO}) ;{ }^{13} \mathrm{C} \mathrm{NMR}: 12.5\left(\mathrm{CH}_{3}\right), 21.3\left(\mathrm{CH}_{2}\right)$, 25.1, 51.6 (pyrrolidino group carbons), 108.1, 125.4, 127.4, 128.3, 139.5, 150.8, 156.6 ( $s p^{2}$ carbons), $182.0(\mathrm{C}=\mathrm{O})$; MS: $\mathrm{m} / \mathrm{z}(\%) 269\left[\mathrm{M}^{+}\right](35)\left(\mathrm{C}_{16} \mathrm{H}_{19} \mathrm{~N}_{3} \mathrm{O}\right), 268[\mathrm{M}-\mathrm{H}](14), 239$ [MCHO-H] (26), 213 [M-2 $\left.\mathrm{C}_{2} \mathrm{H}_{4}\right]$ (13), $143\left[\mathrm{M}-\mathrm{C}_{4} \mathrm{H}_{8} \mathrm{~N}-\mathrm{C}_{3} \mathrm{H}_{5} \mathrm{~N}-\right.$ $\mathrm{H}]$ (19), $91\left[\mathrm{C}_{6} \mathrm{H}_{5} \mathrm{~N}^{+}\right]$(13), $77\left[\mathrm{C}_{6} \mathrm{H}_{5}^{+}\right]$(100), 51 [77- $\left.\mathrm{C}_{2} \mathrm{H}_{2}\right](58)$.

1-Phenyl-3-propyl-5-pyrrolidino- $1 \mathrm{H}$-pyrazole-4carbaldehyde (4e): Fine pale orange cubes, m.p. 94-95 ${ }^{\circ} \mathrm{C}$; Yield $51 \%$; IR (KBr, $\left.v_{\max }, \mathrm{cm}^{-1}\right): 2863(\mathrm{CHO}), 1634(\mathrm{C}=\mathrm{O})$; ${ }^{1} \mathrm{H} \mathrm{NMR}\left(\mathrm{CDCl}_{3}\right): 0.97\left(3 \mathrm{H}, \mathrm{t}, J=7.3, \mathrm{CH}_{3}\right), 1.69(2 \mathrm{H}$, sext, $\left.J=7.3, \mathrm{CH}_{2}\right), 2.77\left(2 \mathrm{H}, \mathrm{t}, J=7.3, \mathrm{CH}_{2}\right), 1.80-1.85(4 \mathrm{H}, \mathrm{m}$, $2 \mathrm{CH}_{2}$ of the pyrrolidino group), 3.21-3.26 $\left(4 \mathrm{H}, \mathrm{m}, \mathrm{CH}_{2}-\mathrm{N}-\right.$ $\left.\mathrm{CH}_{2}\right), 7.31-7.40(5 \mathrm{H}, \mathrm{m}, \mathrm{Ar}-\mathrm{H}), 9.92(1 \mathrm{H}, \mathrm{s}, \mathrm{CHO}) ;{ }^{13} \mathrm{C} \mathrm{NMR}$ : 13.6 $\left(\mathrm{CH}_{3}\right), 21.7\left(\mathrm{CH}_{2}\right), 30.0\left(\mathrm{CH}_{2}\right), 25.1,51.7$ (pyrrolidino group. carbons), 108.4, 125.6, 127.6, 128.5, 139.7, 150.8, 155.6 ( $s p^{2}$ carbons), $182.0(\mathrm{C}=\mathrm{O})$; MS: $m / z(\%) 283\left[\mathrm{M}^{+}\right](41)$ $\left(\mathrm{C}_{17} \mathrm{H}_{21} \mathrm{~N}_{3} \mathrm{O}\right), 282$ [M-H] (30), 254 [M-CHO] (27), 240 [M$\left.\mathrm{C}_{3} \mathrm{H}_{7}\right]$ (36), $227\left[\mathrm{M}-2 \mathrm{C}_{2} \mathrm{H}_{4}\right]$ (39), $144\left[\mathrm{M}-\mathrm{C}_{4} \mathrm{H}_{8} \mathrm{~N}-\mathrm{C}_{4} \mathrm{H}_{7} \mathrm{~N}\right](27)$, $91\left[\mathrm{C}_{6} \mathrm{H}_{5} \mathrm{~N}^{+}\right](21), 77\left[\mathrm{C}_{6} \mathrm{H}_{5}^{+}\right](100), 51\left[77-\mathrm{C}_{2} \mathrm{H}_{2}\right](45)$.
Synthesis of 2-[(3,5-disubstituted 1-phenyl-1H-pyrazol4-yl)methylene]malononitrile (5a-c); 2-cyano-3-(3-ethyl-1phenyl-5-pyrrolidino- $1 H$-pyrazol-4-yl)acrylamide (5d) and 2-cyano-3-(1-phenyl-3-propyl-5-pyrrolidino-1H-pyrazol-4yl)prop-2-enethioamide (5e): A mixture of 4a-e (2.5 mmol) in dry ethanol $(15 \mathrm{~mL})$ was added the active methylene compounds (viz. malonodinitrile, cyanoacetamide and cyanothioacetamide) $(2.5 \mathrm{mmol}$ ) and allowed to reflux for 5-8 h. The progress of the reaction was monitored by TLC. After completion and usual work-up followed by column chromatography gave pure 5a,b,d,e. Compound $\mathbf{5 c}$ was recrystallized from petroleum ether $60-80{ }^{\circ} \mathrm{C}^{25}$.

2-(3-Methyl-5-morpholino-1-phenyl- $1 H$-pyrazol-4ylmethylene)malononitrile (5a): Fine yellow cubes, m.p. 133$144{ }^{\circ} \mathrm{C}$; Yield $71 \%$; IR (KBr, $\left.v_{\max }, \mathrm{cm}^{-1}\right): 2225(\mathrm{CN}), 1583$ $(\mathrm{C}=\mathrm{C}) ;{ }^{1} \mathrm{H}$ NMR $\left(\mathrm{CDCl}_{3}\right): 2.42\left(3 \mathrm{H}, \mathrm{s}, \mathrm{CH}_{3}\right), 2.99(4 \mathrm{H}, \mathrm{t}, \mathrm{J}=$ 4.2, $\left.\mathrm{CH}_{2}-\mathrm{N}-\mathrm{CH}_{2}\right), 3.67$ (4H, t, $\left.J=4.2, \mathrm{CH}_{2}-\mathrm{O}-\mathrm{CH}_{2}\right), 7.45-7.52$ (5H, m, Ar-H), $7.66(1 \mathrm{H}, \mathrm{s}, \mathrm{H}-\mathrm{C}=\mathrm{C}) ;{ }^{13} \mathrm{C} \mathrm{NMR}: 15.1\left(\mathrm{CH}_{3}\right)$, 50.2, 65.9 (morpholino group carbons), 78.2, 106.1, 124.5, 128.5, 128.7, 137.8, 148.6, 150.4, 151.4 ( $s p^{2}$ carbons), 113.0, $114.0(\mathrm{CN})$; MS: $m / z(\%) 319\left[\mathrm{M}^{+}\right]$(44) $\left(\mathrm{C}_{18} \mathrm{H}_{17} \mathrm{~N}_{5} \mathrm{O}\right), 303$ [M-CH $\left.\mathrm{CH}_{3} \mathrm{H}\right]$ (13), 290 [M-HCN-2H] (16), $260\left[\mathrm{M}-\mathrm{C}_{3} \mathrm{H}_{6} \mathrm{O}-\mathrm{H}\right]$ (29), 207 [260-HCN-CN] (11), $91\left[\mathrm{C}_{6} \mathrm{H}_{5} \mathrm{~N}^{+}\right](28), 77\left[\mathrm{C}_{6} \mathrm{H}_{5}{ }^{+}\right]$ (100), $51\left[77-\mathrm{C}_{2} \mathrm{H}_{2}\right]$ (79).

2-(5-Morpholino-1,3-diphenyl-1H-pyrazol-4-ylmethylene)malononitrile (5b): Fine yellow needles, m.p. 177$178{ }^{\circ} \mathrm{C}$; Yield $59 \%$; IR (KBr, $\left.v_{\max }, \mathrm{cm}^{-1}\right): 222(\mathrm{CN}), 1582$ $(\mathrm{C}=\mathrm{C}) ;{ }^{1} \mathrm{H} \mathrm{NMR}\left(\mathrm{CDCl}_{3}\right): 3.06\left(4 \mathrm{H}, \mathrm{t}, J=4.6, \mathrm{CH}_{2}-\mathrm{N}-\mathrm{CH}_{2}\right)$, 3.71 (4H, t, $\left.J=4.6, \mathrm{CH}_{2}-\mathrm{O}-\mathrm{CH}_{2}\right), 7.45-7.56(10 \mathrm{H}, \mathrm{m}, \mathrm{Ar}-\mathrm{H})$, $7.83(1 \mathrm{H}, \mathrm{s}, \mathrm{H}-\mathrm{C}=\mathrm{C}) ;{ }^{13} \mathrm{C} \mathrm{NMR}: 49.5,66.1$ (morpholino group carbons), 82.1, 104.4, 125.2, 127.6, 128.8, 129.0, 131.4, 138.5, 150.4, 151.1, 151.7 ( $s p^{2}$ carbons), 112.1, $114.0(\mathrm{CN})$; MS: $m / z(\%) 381\left[\mathrm{M}^{+}\right](38)\left(\mathrm{C}_{23} \mathrm{H}_{19} \mathrm{~N}_{5} \mathrm{O}\right), 352[\mathrm{M}-\mathrm{HCN}-2 \mathrm{H}](7)$, 324 [352-HCN-H] (9), $322\left[\mathrm{M}-\mathrm{C}_{3} \mathrm{H}_{6} \mathrm{O}-\mathrm{H}\right](14), 91\left[\mathrm{C}_{6} \mathrm{H}_{5} \mathrm{~N}^{+}\right]$ (16), $77\left[\mathrm{C}_{6} \mathrm{H}_{5}^{+}\right]$(100), $51\left[77-\mathrm{C}_{2} \mathrm{H}_{2}\right]$ (49).

2-(1,3-Diphenyl-5-piperidin-1-yl-1H-pyrazol-4ylmethylene)malononitrile (5c): Yellow needles, m.p. 138$139^{\circ} \mathrm{C}$; Yield $99 \%$; IR (KBr, $\left.v_{\max }, \mathrm{cm}^{-1}\right): 2224(\mathrm{CN}), 1578$ $(\mathrm{C}=\mathrm{C}) ;{ }^{1} \mathrm{H}$ NMR $\left(\mathrm{CDCl}_{3}\right): 1.56\left(6 \mathrm{H}\right.$, br. peak, $3 \mathrm{CH}_{2}$ of the piperidino group), 3.05 (4H, br. peak, $\mathrm{CH}_{2}-\mathrm{N}-\mathrm{CH}_{2}$ ), 7.42-7.56 (10H, m, Ar-H), 7.84 (1H,s, H-C=C); ${ }^{13} \mathrm{C}$ NMR: 23.1, 25.3, 51.0 (piperidino group carbons), 77.1, 104.0, 124.9, 127.6, $128.5,128.8,129.0,131.9,138.8,151.3,151.8,152.3\left(s p^{2}\right.$ carbons), 112.3, $114.6(\mathrm{CN})$; MS: $m / z(\%) 379\left[\mathrm{M}^{+}\right]$(52) $\left(\mathrm{C}_{24} \mathrm{H}_{21} \mathrm{~N}_{5}\right), 324$ [M-2HCN-H] (16), 323 [M-2 $\left.\mathrm{C}_{2} \mathrm{H}_{4}\right](8), 246$ $\left[\mathrm{M}-2 \mathrm{C}_{2} \mathrm{H}_{4}-\mathrm{C}_{6} \mathrm{H}_{5}\right](8), 115\left[\mathrm{M}-\mathrm{C}_{4} \mathrm{HN}_{2}-\mathrm{C}_{5} \mathrm{H}_{10} \mathrm{~N}-\mathrm{C}_{7} \mathrm{H}_{5} \mathrm{~N}\right](6), 91$ $\left[\mathrm{C}_{6} \mathrm{H}_{5} \mathrm{~N}^{+}\right](17), 77\left[\mathrm{C}_{6} \mathrm{H}_{5}^{+}\right](100), 51\left[77-\mathrm{C}_{2} \mathrm{H}_{2}\right](41)$.

2-Cyano-3-(3-ethyl-1-phenyl-5-pyrrolidino- $\mathbf{H}$ pyrazol-4-yl)acrylamide (5d): Fine dark yellow cubes, m.p. 183-184 ${ }^{\circ} \mathrm{C}$; Yield $13 \%$; IR ( $\left.\mathrm{KBr}, \nu_{\max }, \mathrm{cm}^{-1}\right)$ : $2209(\mathrm{CN}), 1588$ $(\mathrm{C}=\mathrm{C}), 1674(\mathrm{C}=\mathrm{O}), 3318,3370\left(\mathrm{NH}_{2}\right) ;{ }^{1} \mathrm{H} \mathrm{NMR}\left(\mathrm{CDCl}_{3}\right)$ : $1.25\left(3 \mathrm{H}, \mathrm{t}, J=7.5, \mathrm{CH}_{3}\right), 2.76\left(2 \mathrm{H}\right.$, quint, $\left.J=7.5, \mathrm{CH}_{2}\right), 1.83-$ $1.87\left(4 \mathrm{H}, \mathrm{m}, 2 \mathrm{CH}_{2}\right.$ of the pyrrolidino group), 3.10-3.14 $(4 \mathrm{H}$, $\left.\mathrm{m}, \mathrm{CH}_{2}-\mathrm{N}-\mathrm{CH}_{2}\right), 5.93$ and $6.25(1 \mathrm{H}$, br. s and $1 \mathrm{H}$, br. s, $\left.\mathrm{NH}_{2}\right), 7.33-7.43$ (5H, m, Ar-H), 8.38(1H,s, H-C=C); ${ }^{13} \mathrm{C}$ NMR: $12.2\left(\mathrm{CH}_{3}\right), 21.0\left(\mathrm{CH}_{2}\right), 25.1,50.0$ (pyrrolidino group carbons), 97.3, 103.0, 125.0, 127.5, 128.5, 139.3, 146.1, 148.9, 154.3 ( $s p^{2}$ carbons), $117.3(\mathrm{CN}), 163.1(\mathrm{C}=\mathrm{O})$; MS: $m / z(\%) 335$ 
$\left[\mathrm{M}^{+}\right](62)\left(\mathrm{C}_{19} \mathrm{H}_{21} \mathrm{~N}_{5} \mathrm{O}\right), 291\left[\mathrm{M}-\mathrm{CONH}_{2}\right](80), 252\left[\mathrm{M}-2 \mathrm{C}_{2} \mathrm{H}_{4}{ }^{-}\right.$ $\mathrm{HCN}](43), 222\left[252-\mathrm{C}_{2} \mathrm{H}_{4}-2 \mathrm{H}\right](26), 91\left[\mathrm{C}_{6} \mathrm{H}_{5} \mathrm{~N}^{+}\right](38), 77$ $\left[\mathrm{C}_{6} \mathrm{H}_{5}^{+}\right]$(100), 51 [77- $\left.\mathrm{C}_{2} \mathrm{H}_{2}\right]$ (64).

2-Cyano-3-(1-phenyl-3-propyl-5-pyrrolidino- $\mathbf{H}$ pyrazol-4-yl)prop-2-enethioamide (5e): Fine orange cubes, m.p. 176- $177^{\circ} \mathrm{C}$; Yield 76\%; IR (KBr, $\left.v_{\max }, \mathrm{cm}^{-1}\right)$ : $2204(\mathrm{CN})$, $1645(\mathrm{C}=\mathrm{C}), 1509(\mathrm{C}=\mathrm{S}), 3289,3334\left(\mathrm{NH}_{2}\right) ;{ }^{1} \mathrm{H} \mathrm{NMR}\left(\mathrm{CDCl}_{3}\right)$ : $0.96\left(3 \mathrm{H}, \mathrm{t}, J=7.3, \mathrm{CH}_{3}\right), 1.69\left(2 \mathrm{H}\right.$, sext, $\left.J=7.3, \mathrm{CH}_{2}\right), 2.75$ $\left(2 \mathrm{H}, \mathrm{t}, J=7.3, \mathrm{CH}_{2}\right), 1.80-1.85\left(4 \mathrm{H}, \mathrm{m}, 2 \mathrm{CH}_{2}\right.$ of the pyrrolidino group), 3.21-3.26 (4H, m, $\left.\mathrm{CH}_{2}-\mathrm{N}-\mathrm{CH}_{2}\right), 7.58(2 \mathrm{H}$, br. s, $\left.\mathrm{NH}_{2}\right), 7.38-7.48(5 \mathrm{H}, \mathrm{m}, \mathrm{Ar}-\mathrm{H}), 8.95(1 \mathrm{H}, \mathrm{s}, \mathrm{H}-\mathrm{C}=\mathrm{C}) ;{ }^{13} \mathrm{C} \mathrm{NMR}$ : $13.7\left(\mathrm{CH}_{3}\right), 21.5\left(\mathrm{CH}_{2}\right), 30.5\left(\mathrm{CH}_{2}\right), 25.4,51.1$ (pyrrolidinogroup carbons),101.2, 104.5, 125.5, 128.0, 128.8, 139.6, 150.2, 151.8, 153.6 ( $s p^{2}$ carbons), $116.8(\mathrm{CN}), 193.0(\mathrm{C}=\mathrm{S})$; MS: $m / z(\%) 365\left[\mathrm{M}^{+}\right](25)\left(\mathrm{C}_{20} \mathrm{H}_{23} \mathrm{~N}_{5} \mathrm{~S}\right), 332\left[\mathrm{M}-\mathrm{NH}_{2}-\mathrm{CH}_{3}-2 \mathrm{H}\right]$ (37), 303 [M-CSNH $2-2 \mathrm{H}]$ (78), $266\left[\mathrm{M}-2 \mathrm{C}_{2} \mathrm{H}_{4}-\mathrm{C}_{3} \mathrm{H}_{7}\right]$ (23), 91 $\left[\mathrm{C}_{6} \mathrm{H}_{5} \mathrm{~N}^{+}\right](21), 77\left[\mathrm{C}_{6} \mathrm{H}_{5}^{+}\right]$(100), $51\left[77-\mathrm{C}_{2} \mathrm{H}_{2}\right](48)$.

Synthesis of 3-substituted 1-phenyl-1,4,5a,6,8,9hexahydro-5H-pyrazolo $\left[4^{\prime}, 3^{\prime}: 5,6\right]$ pyrido $[2,1-c][1,4]$ oxazine-5,5-dicarbonitrile (6a,b); 1,3-diphenyl-1,4,6,7,8,9hexahydropyrazolo [4,3 - c] quinolizine - 5,5 (5aH) dicarbonitrile (6c); 5-cyano-3-ethyl-1-phenyl-4,5,5a,6,7,8hexahydro- $1 H$-pyrazolo[3,4-e]indolizine-5-carboxamide (6d) and 5-cyano-1-phenyl-3-propyl-4,5,5a,6,7,8-hexahydro$1 H$-pyrazolo[3,4-e]indolizine-5-carbothioamide (6e): A mixture of $\mathbf{5 a}-\mathbf{e}(2.5 \mathrm{mmol})$ in dry toluene $(15 \mathrm{~mL})$ was added anhydrous zinc chloride $(2.5 \mathrm{mmol})$ and the resulted mixture was refluxed for 7-17 h under anhydrous conditions. After completion, (monitored by TLC) the reaction mixture was cooled, water was added and extracted with dichloromethane $(3 \times 20 \mathrm{~mL})$. The combined organic phases were dried over anhydrous sodium sulfate overnight and concentrated in vacuo. Evaporation of the solvent followed by column chromatography gave pure 6a-e. The compound $\mathbf{6 c}$ was recrystallized from benzene/petroleum ether $60-80^{\circ} \mathrm{C}^{25}$

3-Methyl-1-phenyl-1,4,5a,6,8,9-hexahydro-5Hpyrazolo $\left[4^{\prime}, 3^{\prime}: 5,6\right]$ pyrido[2,1-c] $[1,4]$ oxazine-5,5dicarbonitrile (6a): Pale beige cubes, m.p. $151-152^{\circ} \mathrm{C}$; Yield $36 \%$; IR ( KBr, $\left.\nu_{\max }, \mathrm{cm}^{-1}\right): 2243(\mathrm{CN}) ;{ }^{1} \mathrm{H}$ NMR $\left(\mathrm{CDCl}_{3}\right): 2.21$ $\left(3 \mathrm{H}, \mathrm{s}, \mathrm{CH}_{3}\right), 2.73\left(1 \mathrm{H}, \mathrm{ddd},{ }^{2} J_{9 \mathrm{ax}-9 \mathrm{eq}}=12.1,{ }^{3} J_{9 \mathrm{ax}-8 \mathrm{ax}}=6.8,{ }^{3} J_{9 \mathrm{ax}-8 \mathrm{eq}}\right.$ = 3.4, $\left.\mathrm{H}_{\mathrm{ax}}-9\right), 3.31-3.37[2 \mathrm{H}, \mathrm{m}, \mathrm{H}-5 \mathrm{a},(\mathrm{X}$ part of ABX system $)$ and $\left.\mathrm{H}_{\mathrm{eq}}-9\right], 3.26\left[1 \mathrm{H}, \mathrm{d},{ }^{2} J_{4 \mathrm{ax}-4 \mathrm{eq}}=15.4, \mathrm{H}_{\mathrm{ax}}-4\right.$, (A part of $\mathrm{AB}$ system) $], 3.46\left[1 \mathrm{H}, \mathrm{d},{ }^{2} J_{4 \mathrm{ax}-4 \mathrm{eq}}=15.4, \mathrm{H}_{\mathrm{eq}}-4\right.$, $(\mathrm{B}$ part of $\mathrm{AB}$ system) $], 3.62-3.76\left(2 \mathrm{H}, \mathrm{m}, \mathrm{H}_{2}-8\right), 4.17\left[1 \mathrm{H}, \mathrm{dd},{ }^{2} J_{6 \text { ax-6eq }}=\right.$ $13.5,{ }^{3} J_{5 \mathrm{aax}-6 \mathrm{ax}}=5.9, \mathrm{H}_{\mathrm{ax}}-6$, (A part of ABX system) $], 4.24[1 \mathrm{H}$, $\mathrm{dd},{ }^{2} J_{6 \mathrm{ax}-6 \mathrm{eq}}=13.5,{ }^{3} J_{\text {5aax-6eq }}=3.3, \mathrm{H}_{\mathrm{eq}}-6,($ B part of ABX system $\left.)\right]$, $7.32\left(1 \mathrm{H}, \mathrm{t}, J=7.3, \mathrm{H}^{\prime} 4^{\prime}\right), 7.43$ (2H, t, $\left.J=7.3, \mathrm{H}^{\prime} 3^{\prime}, 5^{\prime}\right), 7.55$ $\left(2 \mathrm{H}, \mathrm{d}, J=7.3, \mathrm{H}-2{ }^{\prime}, 6^{\prime}\right) ;{ }^{13} \mathrm{C}$ NMR: $11.1\left(\mathrm{CH}_{3}\right), 31.2(\mathrm{C}-4)$, 59.0 (C-5a), 66.2 (C-6), 65.0 (C-8), 46.3 (C-9), 112.4, 113.2 (CN), 30.3, 95.1, 143.3, 145.7 (C-5,3a,10a,3),122.5, 126.8, 128.5, 138.6 (phenyl carbons); MS: $m / z$ (\%) $319\left[\mathrm{M}^{+}\right](100)$ $\left(\mathrm{C}_{18} \mathrm{H}_{17} \mathrm{~N}_{5} \mathrm{O}\right), 290$ [M-HCN-2H] (16), 262 [290-HCN-H] (29), $254\left[\mathrm{M}-\mathrm{C}_{3} \mathrm{~N}_{2}-\mathrm{H}\right](26), 198\left[\mathrm{M}-\mathrm{C}_{2} \mathrm{H}_{4} \mathrm{O}-\mathrm{C}_{6} \mathrm{H}_{5}\right](31), 91\left[\mathrm{C}_{6} \mathrm{H}_{5} \mathrm{~N}^{+}\right]$ (26), $77\left[\mathrm{C}_{6} \mathrm{H}_{5}^{+}\right](87), 51\left[77-\mathrm{C}_{2} \mathrm{H}_{2}\right](62)$.

1,3-Diphenyl-1,4,5a,6,8,9-hexahydro-5 $\mathrm{H}$-pyrazolo[4',3':5,6]pyrido[2,1-c][1,4] oxazine-5,5-dicarbonitrile (6b): Pale yellow cubes, m.p. $266-267^{\circ} \mathrm{C}$; Yield $39 \%$; IR (KBr, $\mathrm{v}_{\max }$, $\left.\mathrm{cm}^{-1}\right): 2248(\mathrm{CN}) ;{ }^{1} \mathrm{H} \mathrm{NMR}\left(\mathrm{CDCl}_{3}\right): 2.79\left(1 \mathrm{H}, \mathrm{ddd},{ }^{2} J_{9 \mathrm{ax}-9 \mathrm{eq}}=\right.$ $\left.12.0,{ }^{3} J_{9 \mathrm{ax}-8 \mathrm{ax}}=6.0,{ }^{3} J_{9 \mathrm{ax}-8 \mathrm{eq}}=3.3, \mathrm{H}_{\mathrm{ax}}-9\right), 3.40\left(1 \mathrm{H}, \mathrm{ddd},{ }^{2} J_{9 \mathrm{ax}-9 \mathrm{eq}}=\right.$ $\left.12.0,{ }^{3} J_{9 \mathrm{ax}-8 \mathrm{ax}}=6.0,{ }^{3} J_{9 \mathrm{ax}-8 \mathrm{eq}}=3.7, \mathrm{H}_{\mathrm{eq}}-9\right), 3.45(1 \mathrm{H}, \mathrm{t}, J=5.8, \mathrm{H}-$ $5 \mathrm{a}), 3.57\left[1 \mathrm{H}, \mathrm{d},{ }^{2} J_{4 a x-4 \mathrm{eq}}=15.4, \mathrm{H}_{\mathrm{ax}}-4\right.$, (A part of AB system) $]$, $3.72\left[1 \mathrm{H}, \mathrm{d},{ }^{2} J_{4 \mathrm{ax}-4 \mathrm{eq}}=15.4, \mathrm{H}_{\mathrm{eq}}-4,(\mathrm{~B}\right.$ part of $\mathrm{AB}$ system $)$, 3.67-3.77 (2H, m, $\left.\mathrm{H}_{2}-8\right), 4.26\left(2 \mathrm{H}, \mathrm{d}, J=5.1, \mathrm{H}_{2}-6\right)$, 7.357.51 (6H, m, H-3',3', ,4',4', 5'.5'), 7.65 (4H, d, J = 7.0, H2',2', 6',6'"); ${ }^{13} \mathrm{C}$ NMR: 32.8 (C-4), 59.0 (C-5a), 66.4 (C-6), 65.1 (C-8), 46.4 (C-9), 112.9, 113.4 (CN), 30.5, 94.5, 144.1, 148.3 (C-5,3a,10a,3), 122.9, 126.1, 127.2, 127.7, 128.1, 128.8, 131.4, 138.8 (phenyl group carbons); MS: $m / z(\%) 381\left[\mathrm{M}^{+}\right]$ (60) $\left(\mathrm{C}_{23} \mathrm{H}_{19} \mathrm{~N}_{5} \mathrm{O}\right), 352$ [M-HCN-2H] (11), 324 [352-HCN-H] (4), $316\left[\mathrm{M}-\mathrm{C}_{3} \mathrm{~N}_{2}-\mathrm{H}\right]$ (6), $260\left[\mathrm{M}-\mathrm{C}_{2} \mathrm{H}_{4} \mathrm{O}-\mathrm{C}_{6} \mathrm{H}_{5}\right]$ (5), 91 $\left[\mathrm{C}_{6} \mathrm{H}_{5} \mathrm{~N}^{+}\right](10), 77\left[\mathrm{C}_{6} \mathrm{H}_{5}^{+}\right]$(100), 51 [77- $\left.\mathrm{C}_{2} \mathrm{H}_{2}\right]$ (46).

1,3-Diphenyl-1,4,6,7,8,9-hexahydropyrazolo $[4,3$ c]quinolizine-5,5(5aH)-dicarbonitrile (6c): White powder, m.p. 209-210 ${ }^{\circ}$; Yield $21 \%$; IR (KBr, $\left.v_{\max }, \mathrm{cm}^{-1}\right)$ : $2253(\mathrm{CN})$; ${ }^{1} \mathrm{H}$ NMR $\left(\mathrm{CDCl}_{3}\right): 1.54$ (3H, br. peak, $\left.\mathrm{H}_{2}-7, \mathrm{H}_{\mathrm{ax}}-8\right), 1.86-2.05$ [2H, m, $\mathrm{H}_{\mathrm{ax}}-6$, (A part of ABX system) and $\left.\mathrm{H}_{\mathrm{eq}}-8\right], 2.33$ [1H, $\mathrm{d},{ }^{2} J_{6 \mathrm{ax}-6 \mathrm{eq}}=12.4, \mathrm{H}_{\mathrm{eq}}-6$, (B part of ABX system) $], 2.47-2.55$ $\left(1 \mathrm{H}, \mathrm{m}, \mathrm{H}_{\mathrm{ax}}-9\right), 3.28\left[1 \mathrm{H}, \mathrm{dd},{ }^{3} J_{5 \text { aax-6ax }}=9.0,{ }^{3} J_{5 \text { aax-6eq }}=2.5, \mathrm{H}-\right.$ 5a, (X part of ABX system) ], $3.43\left(1 \mathrm{H}, \mathrm{d},{ }^{2} J_{9 \mathrm{ax}-9 \mathrm{eq}}=11.5, \mathrm{H}_{\mathrm{eq}}-9\right)$, $3.53\left[1 \mathrm{H}, \mathrm{d},{ }^{2} J_{4 \mathrm{ax}-4 \mathrm{eq}}=15.1, \mathrm{H}_{\mathrm{ax}}-4\right.$, (A part of AB system) $]$, $3.61\left[1 \mathrm{H}, \mathrm{d},{ }^{2} J_{4 \mathrm{ax}-4 \mathrm{eq}}=15.1, \mathrm{H}_{\mathrm{eq}}-4\right.$, (B part of AB system)], 7.33-7.50 (6H, m, H-3',3', 4',4', 5'.5'), 7.62 (4H, d, $J=7.0$, H-2',2', 6',6'); ${ }^{13} \mathrm{C}$ NMR: 32.2 (C-4), 62.6 (C-5a), 23.3 (C-6), 22.3 (C-7), 27.5 (C-8), 49.7 (C-9), 112.7, 113.9 (CN), 36.6, 93.9, 145.0, 148.6 (C-5,3a,10a,3),123.1, 126.4, 127.2, 127.7, 128.1, 128.7, 131.6, 139.6 (phenyl group carbons); MS: $\mathrm{m} / \mathrm{z}$ (\%) $379\left[\mathrm{M}^{+}\right](100)\left(\mathrm{C}_{24} \mathrm{H}_{21} \mathrm{~N}_{5}\right), 351[\mathrm{M}-\mathrm{HCN}-\mathrm{H}](10), 325$ $[\mathrm{M}-2 \mathrm{HCN}](5), 314\left[\mathrm{M}-\mathrm{C}_{3} \mathrm{~N}_{2}-\mathrm{H}\right]$ (37), $246\left[\mathrm{M}-2 \mathrm{C}_{2} \mathrm{H}_{4}-\mathrm{C}_{6} \mathrm{H}_{5}\right]$ (16), $115\left[\mathrm{M}-\mathrm{C}_{7} \mathrm{H}_{5} \mathrm{~N}-\mathrm{C}_{9} \mathrm{H}_{11} \mathrm{~N}_{3}\right](23), 91\left[\mathrm{C}_{6} \mathrm{H}_{5} \mathrm{~N}^{+}\right](12), 77\left[\mathrm{C}_{6} \mathrm{H}_{5}{ }^{+}\right]$ (92), $51\left[77-\mathrm{C}_{2} \mathrm{H}_{2}\right](52)$.

5-Cyano-3-ethyl-1-phenyl-4,5,5a,6,7,8-hexahydro- $1 \mathrm{H}$ pyrazolo[3,4-e]indolizine-5-carboxamide (6d): Yellow scales, m.p. 204-206 ${ }^{\circ} \mathrm{C}$; Yield $31 \%$; IR $\left(\mathrm{KBr}, \mathrm{v}_{\max }, \mathrm{cm}^{-1}\right)$ : $2229(\mathrm{CN}), 1692(\mathrm{C}=\mathrm{O}), 3305,3386\left(\mathrm{NH}_{2}\right)$; ${ }^{1} \mathrm{H} \mathrm{NMR}\left(\mathrm{CDCl}_{3}\right)$ : $0.83\left(3 \mathrm{H}, \mathrm{t}, J=8.1, \mathrm{CH}_{3}\right), 2.61$ ( $2 \mathrm{H}$, quint, $\left.J=8.1, \mathrm{CH}_{2}\right), 1.86-$ $2.11\left(3 \mathrm{H}, \mathrm{m}, \mathrm{H}_{\mathrm{ax}}-6, \mathrm{H}_{2}-7\right), 2.32-2.37\left(1 \mathrm{H}, \mathrm{m}, \mathrm{H}_{\mathrm{eq}}-6\right), 2.91-3.00$ (1H, m, $\left.\mathrm{H}_{\mathrm{ax}}-8\right), 3.07-3.20\left(3 \mathrm{H}, \mathrm{m}, \mathrm{H}_{2}-4, \mathrm{H}_{\mathrm{eq}}-8\right), 3.97(1 \mathrm{H}, \mathrm{dd}$, $\left.{ }^{3} J_{5 \text { aаx-6ax }}=7.7,{ }^{3} J_{5 \text { aax-6eq }}=4.4, \mathrm{H}-5 \mathrm{a}\right), 5.80$ and $6.53(1 \mathrm{H}$, br. $\mathrm{s}$ and1H, br. s, $\mathrm{D}_{2} \mathrm{O}$ exchangeable, $\left.\mathrm{NH}_{2}\right), 7.29(1 \mathrm{H}, \mathrm{t}, J=7.9, \mathrm{H}-$ $\left.4^{\prime}\right), 7.41\left(2 \mathrm{H}, \mathrm{t}, J=7.9, \mathrm{H}^{-} 3^{\prime}, 5^{\prime}\right), 7.53$ (2H, d, $\left.J=7.9, \mathrm{H}-2^{\prime}, 6^{\prime}\right)$; ${ }^{13} \mathrm{C}$ NMR: $12.3\left(\mathrm{CH}_{3}\right), 21.7\left(\mathrm{CH}_{2}\right), 36.1(\mathrm{C}-4), 63.4(\mathrm{C}-5 \mathrm{a})$, 22.9 (C-6), 27.0 (C-7), 43.3 (C-8), 48.9, 93.8, 143.0, 150.9 (C-5,3a,9a,3),123.3, 126.0, 128.0,138.8 (phenyl group carbons), $119.2(\mathrm{CN}), 168.1(\mathrm{C}=\mathrm{O})$; MS: $\mathrm{m} / z(\%) 335\left[\mathrm{M}^{+}\right]$ (62) $\left(\mathrm{C}_{19} \mathrm{H}_{21} \mathrm{~N}_{5} \mathrm{O}\right), 291$ [M-CONH$\left.H_{2}\right](96), 252$ [M-2 $\left.\mathrm{C}_{2} \mathrm{H}_{4}-\mathrm{HCN}\right]$ (12), $248\left[291-\mathrm{C}_{2} \mathrm{H}_{4}-\mathrm{CH}_{3}\right](13), 91\left[\mathrm{C}_{6} \mathrm{H}_{5} \mathrm{~N}^{+}\right](17), 77\left[\mathrm{C}_{6} \mathrm{H}_{5}{ }^{+}\right]$ (100), 51 [77- $\left.\mathrm{C}_{2} \mathrm{H}_{2}\right](47)$.

5-Cyano-1-phenyl-3-propyl-4,5,5a,6,7,8-hexahydro$1 \mathrm{H}$-pyrazolo[3,4-e]indolizine-5-carbothioamide (6e): Orange cubes, m.p. $186{ }^{\circ} \mathrm{C}$; Yield $46 \%$; IR $\left(\mathrm{KBr}, v_{\max }, \mathrm{cm}^{-1}\right)$ : $2234(\mathrm{CN}), 1526(\mathrm{C}=\mathrm{S}), 3358,3423\left(\mathrm{NH}_{2}\right) ;{ }^{1} \mathrm{H} \mathrm{NMR}\left(\mathrm{CDCl}_{3}\right)$ : $0.97\left(3 \mathrm{H}, \mathrm{t}, J=7.3, \mathrm{CH}_{3}\right), 1.67\left(2 \mathrm{H}\right.$, sext, $\left.J=7.3, \mathrm{CH}_{2}\right), 2.55$ $\left(2 \mathrm{H}, \mathrm{t}, J=7.3, \mathrm{CH}_{2}\right), 1.81-1.94\left(2 \mathrm{H}, \mathrm{m}, \mathrm{H}_{\mathrm{ax}}-6, \mathrm{H}_{\mathrm{ax}}-7\right), 2.01-$ $2.06\left(1 \mathrm{H}, \mathrm{m}, \mathrm{H}_{\mathrm{eq}}-7\right), 2.27-2.38\left(1 \mathrm{H}, \mathrm{m}, \mathrm{H}_{\mathrm{eq}}-6\right), 2.93-3.02(1 \mathrm{H}$, $\left.\mathrm{m}, \mathrm{H}_{\mathrm{ax}}-8\right), 3.05\left[1 \mathrm{H}, \mathrm{d},{ }^{2} J_{4 \mathrm{ax}-4 \mathrm{eq}}=15.4, \mathrm{H}_{\mathrm{ax}}-4\right.$, (A part of $\mathrm{AB}$ system)], 3.17-3.23 (1H, m, Heq-8), 3.49[1H, d ${ }^{2} J_{4 a x-4 e q}=15.4$, $\mathrm{H}_{\mathrm{eq}}-4$, (B part of AB system)], $4.22\left(1 \mathrm{H}, \mathrm{dd},{ }^{3} J_{5 \text { aax-6ax }}=6.0\right.$, $\left.{ }^{3} J_{5 \text { aax-6eq }}=4.8, \mathrm{H}-5 \mathrm{a}\right), 7.28(1 \mathrm{H}, \mathrm{t}, J=7.6, \mathrm{H}-4), 7.41(2 \mathrm{H}, \mathrm{t}$, 
$\left.J=7.6, \mathrm{H}-3^{\prime}, 5^{\prime}\right), 7.52\left(2 \mathrm{H}, \mathrm{d}, J=7.6, \mathrm{H}-2^{\prime}, 6^{\prime}\right), 7.81$ and8.81 $\left(1 \mathrm{H}\right.$, br. s and $1 \mathrm{H}$, br. s $\mathrm{D}_{2} \mathrm{O}$ exchangeable, $\left.\mathrm{NH}_{2}\right) ;{ }^{13} \mathrm{C}$ NMR: $13.4\left(\mathrm{CH}_{3}\right), 21.8\left(\mathrm{CH}_{2}\right), 28.8\left(\mathrm{CH}_{2}\right), 33.5(\mathrm{C}-4), 67.2(\mathrm{C}-5 \mathrm{a})$, 23.1 (C-6), 27.0 (C-7), 49.4 (C-8), 50.2, 95.4, 143.1, 150.0 (C-5,3a,9a,3),123.7, 126.4, 128.3,139.1 (phenyl group carbons), $119.0(\mathrm{CN}), 201.3(\mathrm{C}=\mathrm{S}) ; \mathrm{MS}: \mathrm{m} / z(\%) 365\left[\mathrm{M}^{+}\right](70)$ $\left(\mathrm{C}_{20} \mathrm{H}_{23} \mathrm{~N}_{5} \mathrm{~S}\right), 332$ [M-NH$\left.{ }_{2}-\mathrm{CH}_{3}-2 \mathrm{H}\right](67), 303\left[\mathrm{M}-\mathrm{CSNH}_{2}-2 \mathrm{H}\right]$ (100), $267\left[\mathrm{M}-\mathrm{C}_{3} \mathrm{H}_{2} \mathrm{~N}_{2} \mathrm{~S}\right](11), 91\left[\mathrm{C}_{6} \mathrm{H}_{5} \mathrm{~N}^{+}\right]$(36), $77\left[\mathrm{C}_{6} \mathrm{H}_{5}{ }^{+}\right]$ (94), 51 [77- $\left.\mathrm{C}_{2} \mathrm{H}_{2}\right]$ (42).

Synthesis of 2-arylidenemalononitriles (8): They were synthesized by reaction of aromatic aldehyde with malononitrile according to the literature procedures ${ }^{26}$.

Synthesis of 6-amino-1,4-disubstituted 3-propyl-1,4dihydro-pyrano[2,3-c]pyrazole-5-carbonitrile (9a-c): They were synthesized by following different methods.

Method A: They were synthesized using the reported method $^{27}$.

Method B: A mixture of 2e,f $(1.25 \mathrm{mmol})$, aromatic aldehyde 7a-c $(1.25 \mathrm{mmol})$, malononitrile $(1.25 \mathrm{mmol})$ and piperidine (few drops) was placed in a $100 \mathrm{~mL}$ beaker covered with a watch glass and was then irradiated with microwave (for $5 \mathrm{~min}$ at $300 \mathrm{~W}$ for $9 \mathbf{b}$ and $15 \mathrm{~min}$ at $300 \mathrm{~W}$ then $15 \mathrm{~min}$ at $400 \mathrm{~W}$ for 9a,c), (monitored by TLC) the solid product obtained after cooling was collected by filtration, washed with ethanol to give pure 9a. The cold reaction mixture was treated with ethyl acetate or ether, the solid product was filtered and recrystallized from water/ethanol to give $\mathbf{9 b}$, the solid product was then subjected to column chromatography to give pure $9 c$.

Method C: A mixture of 2e,f(1.25 mmol), 7a-c(1.25 mmol), malononitrile $(1.25 \mathrm{mmol})$ and cetyltrimethylammonium chloride $(\mathrm{CTACl})$ in water $(10 \mathrm{~mL})$ was placed in a $25 \mathrm{~mL}$ conical flask and was then irradiated in water bath of an ultrasonic cleaner for $1.5 \mathrm{~h}$, the solid product was filtered, washed with water to give pure 9a. The reaction mixture was extracted with dichloromethane $(3 \times 20 \mathrm{~mL})$. The combined organic phases were concentrated in vacuo and treated with ethyl acetate then with ether, the solid product was filtered and recrystallized from water/ethanol to give $\mathbf{9 b}$ and thesolid product was subjected to column chromatography to give pure $9 \mathrm{c}$.

6-Amino-3-propyl-1-(2-pyridinyl)-4-(3',4',5'-trimethoxyphenyl)-1,4-dihydro-pyrano[2,3-c]pyrazole-5-carbonitrile (9a): White fine needles, m.p. $162-163^{\circ} \mathrm{C}$; Yield $79^{\mathrm{A}}, 88^{\mathrm{B}}$, $51^{\mathrm{C}} \%$; IR (KBr, $\left.v_{\max }, \mathrm{cm}^{-1}\right): 2198(\mathrm{CN}), 3323,3466\left(\mathrm{NH}_{2}\right) ;{ }^{1} \mathrm{H}$ NMR (DMSO- $\left.d_{6}\right)$ : $0.72\left(3 \mathrm{H}, \mathrm{t}, J=7.3, \mathrm{CH}_{3} \mathrm{CH}_{2}\right), 1.10-1.24$ $(1 \mathrm{H}, \mathrm{m}), 1.27-1.44(1 \mathrm{H}, \mathrm{m}),\left(\mathrm{CH}_{2}\right.$ of the propyl group near the chiral carbon C-4), 2.08-2.23 (2H, m, $\left.\mathrm{CH}_{3} \mathrm{CH}_{2} \mathrm{CH}_{2}\right), 3.64(3 \mathrm{H}$, $\left.\mathrm{s}, \mathrm{OCH}_{3}\right), 3.72\left(6 \mathrm{H}, \mathrm{s}, 2 \mathrm{OCH}_{3}\right), 4.66(1 \mathrm{H}, \mathrm{s}, \mathrm{H}-4), 6.55(2 \mathrm{H}, \mathrm{s}$, H-2", 6" ) 7.08 (2H, br.s, $\left.\mathrm{NH}_{2}\right)\left(\mathrm{D}_{2} \mathrm{O}\right.$ exchangeable), $7.36(1 \mathrm{H}$, $\left.\mathrm{dd},{ }^{3} J_{4,5}=7.7,{ }^{3} J_{5,6}=5.1, \mathrm{H}-5{ }^{\prime}\right), 7.71\left(1 \mathrm{H}, \mathrm{d},{ }^{3} J_{3,4}=7.7, \mathrm{H}-\right.$ $\left.3^{\prime}\right), 7.96\left(1 \mathrm{H}, \mathrm{td},{ }^{3} \mathrm{~J}=7.7,{ }^{4} J_{4,6}=1.8, \mathrm{H}-4\right), 8.49\left(1 \mathrm{H}, \mathrm{d},{ }^{3} J_{5,6}=\right.$ 5.1, H-6'); ${ }^{13} \mathrm{C}$ NMR: $13.6\left(\mathrm{CH}_{3}\right), 20.6\left(\mathrm{CH}_{3} \mathrm{CH}_{2}\right), 28.8$ $\left(\mathrm{CH}_{2} \mathrm{CH}_{2}\right), 36.9(\mathrm{C}-4), 55.8\left(2 \mathrm{OCH}_{3}\right), 59.9\left(\mathrm{OCH}_{3}\right), 119.8$ (CN),115.1 (C-3'), 121.9 (C-5'), 138.9 (C-4'), 148.1 (C-6'), 57.9, 98.6, 104.9, 136.3, 139.4, 144.2, 149.9, 150.3, 152.7, 159.4 ( $s p^{2}$ carbons); MS (ESI): $m / z(\%) 448[\mathrm{M}+\mathrm{H}]^{+}(100)$ $\left(\mathrm{C}_{24} \mathrm{H}_{25} \mathrm{~N}_{5} \mathrm{O}_{4}+\mathrm{H}\right), 430\left[\mathrm{M}+\mathrm{H}-\mathrm{NH}_{3}-\mathrm{H}\right](5), 382\left[\mathrm{M}+\mathrm{H}-\mathrm{C}_{3} \mathrm{H}_{2} \mathrm{~N}_{2}\right]$ (60), $280\left[\mathrm{M}+\mathrm{H}-\mathrm{C}_{9} \mathrm{H}_{11} \mathrm{O}_{3}-\mathrm{H}\right]$ (55), 251 [280- $\left.\mathrm{C}_{2} \mathrm{H}_{5}\right]$ (12).

6-Amino-1-methyl-3-propyl-4-(2-thienyl)-1,4-dihydropyrano[2,3-c]pyrazole-5-carbonitrile $(9 \mathrm{~b})$ : Pale green cubes, m.p. $166-168^{\circ} \mathrm{C}$; Yield $25^{\mathrm{A}}, 26^{\mathrm{B}}, 8^{\mathrm{C}} \%$; IR $\left(\mathrm{KBr}, \mathrm{v}_{\max }, \mathrm{cm}^{-1}\right)$ : $2189(\mathrm{CN}), 3314,3380\left(\mathrm{NH}_{2}\right) ;{ }^{1} \mathrm{H}$ NMR (DMSO-d $)$ : $0.91(3 \mathrm{H}$, $\left.\mathrm{t}, J=7.3, \mathrm{CH}_{3}\right), 1.54\left(2 \mathrm{H}\right.$, sext, $\left.J=7.3, \mathrm{CH}_{2}\right), 2.45-2.50(2 \mathrm{H}$, $\mathrm{m}, \mathrm{CH}_{2}$ within $\mathrm{H}_{2} \mathrm{O}$ in DMSO, $\left(2.52,2 \mathrm{H}, \mathrm{t}, J=7.3\right.$ in $\left.\mathrm{CDCl}_{3}\right)$ ), $3.36\left(3 \mathrm{H}\right.$, br s, $\mathrm{N}-\mathrm{CH}_{3}$ with DMSO, $\left(3.45,3 \mathrm{H}\right.$, s, in $\left.\mathrm{CDCl}_{3}\right)$ ), $4.88(1 \mathrm{H}, \mathrm{s}, \mathrm{H}-4), 6.54\left(2 \mathrm{H}, \mathrm{d},{ }^{3} J_{3,4}=3.5, \mathrm{H}-3{ }^{\prime}\right), 6.82(1 \mathrm{H}, \mathrm{dd}$, $\left.{ }^{3} J_{4,5}=5.0,{ }^{3} J_{3,4}=3.5, \mathrm{H}-4^{\prime}\right), 7.20\left(1 \mathrm{H}, \mathrm{d},{ }^{3} J_{4,5}=5.0, \mathrm{H}-5^{\prime}\right)$, 14.0(2H, br.s, $\left.\mathrm{NH}_{2}\right)\left(\mathrm{D}_{2} \mathrm{O}\right.$ exchangeable); $\mathrm{MS}(\mathrm{ESI}): \mathrm{m} / \mathrm{z}(\%)$ $301[\mathrm{M}+\mathrm{H}]^{+}$(not observed) $\left(\mathrm{C}_{15} \mathrm{H}_{16} \mathrm{~N}_{4} \mathrm{OS}+\mathrm{H}\right), 235[\mathrm{M}+\mathrm{H}-$ $\left.\mathrm{C}_{3} \mathrm{H}_{2} \mathrm{~N}_{2}\right]$ (90), $141\left[\mathrm{M}+\mathrm{H}-\mathrm{C}_{8} \mathrm{H}_{6} \mathrm{~N}_{2} \mathrm{~S}+2 \mathrm{H}\right]$ (100).

6-Amino-4-(2-furyl)-1-methyl-3-propyl-1,4-dihydropyrano[2,3-c]pyrazole-5-carbonitrile (9c): Pale green cubes, m.p. $166-168^{\circ} \mathrm{C}$; Yield $25^{\mathrm{A}}, 26^{\mathrm{B}}, 8^{\mathrm{C}} \%$; IR $\left(\mathrm{KBr}, v_{\max }, \mathrm{cm}^{-1}\right)$ : 2209 (CN), 3355, $3414\left(\mathrm{NH}_{2}\right)$; MS (ESI): $m / z(\%) 285[\mathrm{M}+\mathrm{H}]^{+}$ (30) $\left(\mathrm{C}_{15} \mathrm{H}_{16} \mathrm{~N}_{4} \mathrm{O}_{2}+\mathrm{H}\right), 268\left[\mathrm{M}+\mathrm{H}-\mathrm{NH}_{3}\right]$ (100), $240[\mathrm{M}+\mathrm{H}-$ $\mathrm{HCN}-\mathrm{H}]$ (20), 151 [M+H- $\left.\mathrm{C}_{3} \mathrm{H}_{2} \mathrm{~N}_{2}-\mathrm{C}_{4} \mathrm{H}_{3} \mathrm{O}-\mathrm{H}\right]$ (5), 99 [M+H$\left.\mathrm{C}_{10} \mathrm{H}_{6} \mathrm{~N}_{2} \mathrm{O}_{2}\right]$ (4).

Synthesis of 1,4-disubstituted-7-Methyl-3-propyl-4,6dihydropyrazolo $\left[4^{\prime}, 3^{\prime}: 5,6\right]$ pyrano $[2,3-d]$ pyrimidin-5(1H)one $(\mathbf{1 1 a}, \mathbf{b})$ : They were synthesized using the reported method $^{26}$

7-Methyl-3-propyl-1-(2-pyridinyl)-4-(3,4,5-trimethoxyphenyl)-4,6-dihydropyrazolo $\left[4^{\prime}, 3\right.$ ':5,6]pyrano[2,3-d]pyrimidin-5(1H)-one (11a): Pale yellow powder, m.p. $>300{ }^{\circ} \mathrm{C}$ (from ethanol); Yield 57\%; IR $\left(\mathrm{KBr}, \mathrm{v}_{\max }, \mathrm{cm}^{-1}\right): 3449$ $(\mathrm{NH}), 1655(\mathrm{C}=\mathrm{O}) ;{ }^{1} \mathrm{H}$ NMR (DMSO- $\left.d_{6}\right): 0.78(3 \mathrm{H}, \mathrm{t}, J=7.3$, $\left.\mathrm{CH}_{3}\right), 1.17-1.29(1 \mathrm{H}, \mathrm{m}), 1.35-1.46(1 \mathrm{H}, \mathrm{m})\left(\mathrm{CH}_{2}\right.$ of the propyl group near the chiral carbon $\mathrm{C}-4), 2.29-2.34\left(5 \mathrm{H}, \mathrm{m}, \mathrm{CH}_{2}\right.$, $\mathrm{CH}_{3}$ at C-7), $3.60\left(3 \mathrm{H}, \mathrm{s}, \mathrm{OCH}_{3}\right), 3.69\left(6 \mathrm{H}, \mathrm{s}, 2 \mathrm{OCH}_{3}\right), 5.01$ (1H, s, H-4), 6.56 (2H, s, H-2', 6'"), $7.36\left(1 \mathrm{H}, \mathrm{ddd},{ }^{3} J_{4,5}=7.9\right.$, $\left.{ }^{3} J_{5,6}=4.9,{ }^{4} J_{3,5}=1.1, \mathrm{H}-5^{\prime}\right), 7.72\left(1 \mathrm{H}, \mathrm{d},{ }^{3} J_{3,4}=7.9, \mathrm{H}-3^{\prime}\right), 7.97$ $\left(1 \mathrm{H}, \mathrm{td},{ }^{3} J=7.9,{ }^{4} J_{4,6}=1.8, \mathrm{H}-4\right), 8.52\left(1 \mathrm{H}, \mathrm{dd},{ }^{3} J_{5,6}=4.9,{ }^{4} J_{4,6}\right.$ $=1.8, \mathrm{H}-6)$ ') ${ }^{13} \mathrm{C}$ NMR: 13.6, 20.7, 20.8, 28.9, 34.8 (Pr carbons, $\mathrm{CH}_{3}$ at C-7, C-4), $55.7\left(2 \mathrm{OCH}_{3}\right), 59.8\left(\mathrm{OCH}_{3}\right), 100.3,100.4$, $105.4,114.7,121.7,136.0,138.9,139.9,145.1,148.1,150.3$, 150.6, 152.4, 158.5, 160.7 ( $s p^{2}$ carbons), $162.6(\mathrm{C}=\mathrm{O})$; MS (ESI): $m / z(\%) 490[\mathrm{M}+\mathrm{H}]^{+}(25)\left(\mathrm{C}_{26} \mathrm{H}_{27} \mathrm{~N}_{5} \mathrm{O}_{5}+\mathrm{H}\right), 460[\mathrm{M}+\mathrm{H}-$ $\left.\mathrm{C}_{2} \mathrm{H}_{5}-\mathrm{H}\right]$ (5), $382\left[\mathrm{M}+\mathrm{H}-\mathrm{C}_{5} \mathrm{H}_{4} \mathrm{~N}_{2} \mathrm{O}\right](5), 322\left[\mathrm{M}+\mathrm{H}-\mathrm{C}_{9} \mathrm{H}_{11} \mathrm{O}_{3}-\right.$ $\mathrm{H}]$ (100), 281 [280- $\left.\mathrm{CH}_{3}-\mathrm{HCN}-\mathrm{H}\right](75)$.

1,7-Dimethyl-3-propyl-4-(2-thienyl)-4,6-dihydropyrazolo $\left[4^{\prime}, 3^{\prime}: 5,6\right]$ pyrano $[2,3-d]$ pyrimidin-5(1H)-one (11b): Pale green powder, m.p. $>300^{\circ} \mathrm{C}$; Yield $38 \%$; IR (KBr, $\left.v_{\max }, \mathrm{cm}^{-1}\right): 3484(\mathrm{NH}), 1625(\mathrm{C}=\mathrm{O}) ;{ }^{1} \mathrm{H}$ NMR (DMSO- $\left.d_{6}\right)$ : $0.93\left(3 \mathrm{H}, \mathrm{t}, J=7.1, \mathrm{CH}_{3}\right), 1.61-1.54\left(2 \mathrm{H}, \mathrm{m}, \mathrm{CH}_{2}\right), 2.73-2.66$ $\left(2 \mathrm{H}, \mathrm{m}, \mathrm{CH}_{2}\right), 2.50\left(3 \mathrm{H}, \mathrm{s}, \mathrm{CH}_{3}\right.$ at $\left.\mathrm{C}-7\right), 3.52\left(3 \mathrm{H}, \mathrm{s}, \mathrm{N}-\mathrm{CH}_{3}\right)$, 5.15 (1H, s, H-4), 6.39 (1H, d, ${ }^{3} J_{3,4}=3.4, \mathrm{H}-3$ '), 6.87-6.85 (2H, m, H-4',5'); ${ }^{13} \mathrm{C}$ NMR: 12.8, 21.4, 25.1, 27.7, 30.6, 33.0 (Pr carbons, $\mathrm{CH}_{3}$ at $\left.\mathrm{C}-7, \mathrm{~N}-\mathrm{CH}_{3}, \mathrm{C}-4\right), 103.4,122.4,124.3$, 127.0, 128.9, 144.9, 145.7, 149.2, 152.0, 157.4 ( $s p^{2}$ carbons), $159.5(\mathrm{C}=\mathrm{O}) ; \mathrm{MS}(\mathrm{ESI}): \mathrm{m} / z(\%) 343[\mathrm{M}+\mathrm{H}]^{+}$(not observed) $\left(\mathrm{C}_{17} \mathrm{H}_{18} \mathrm{~N}_{4} \mathrm{O}_{2} \mathrm{~S}+\mathrm{H}\right), 315\left[\mathrm{M}+\mathrm{H}-\mathrm{C}_{2} \mathrm{H}_{4}\right](75), 233[\mathrm{M}+\mathrm{H}-$ $\left.\mathrm{C}_{5} \mathrm{H}_{4} \mathrm{~N}_{2} \mathrm{O}-2 \mathrm{H}\right](2), 141\left[\mathrm{M}+\mathrm{H}-\mathrm{C}_{10} \mathrm{H}_{8} \mathrm{~N}_{2} \mathrm{OS}+2 \mathrm{H}\right]$ (100).

\section{RESULTS AND DISCUSSION}

The starting materials, namely pyrazolones $\mathbf{2 a - f}$ were synthesized by the reactions of the appropriate hydrazine with $\beta$-ketoesters using microwave and ultrasound irradiation according to literature procedure ${ }^{23}$ (Scheme-I). 5-Chloro-3substituted 1-phenyl-1H-pyrazole-4-carbaldehyde 3a-d were 
<smiles>[R]C(=O)CC(C)=O</smiles>

3a-d<smiles>[R]C1=NN([R])C(=O)C1</smiles>

2a-f

\begin{tabular}{|c|c|c|}
\hline & $\underline{\mathbf{R}}$ & $\underline{\mathbf{R}}^{1}$ \\
\hline $\mathbf{a}$ & $\mathrm{CH}_{3}$ & $\mathrm{C}_{6} \mathrm{H}_{5}$ \\
\hline b & $\mathrm{C}_{6} \mathrm{H}_{5}$ & $\mathrm{C}_{6} \mathrm{H}_{5}$ \\
\hline c & $\mathrm{C}_{2} \mathrm{H}_{5}$ & $\mathrm{C}_{6} \mathrm{H}_{5}$ \\
\hline d & $\mathrm{C}_{3} \mathrm{H}_{7}$ & $\mathrm{C}_{6} \mathrm{H}_{5}$ \\
\hline $\mathbf{e}$ & $\mathrm{C}_{3} \mathrm{H}_{7}$ & 2-pyridinyl \\
\hline $\mathbf{f}$ & $\mathrm{C}_{3} \mathrm{H}_{7}$ & $\mathrm{CH}_{3}$ \\
\hline
\end{tabular}

R

$\mathrm{CH}_{3}$

$\mathrm{C}_{6} \mathrm{H}_{5}$

$\mathrm{C}_{6} \mathrm{H}_{5}$

$\mathrm{C}_{2} \mathrm{H}_{5}$

$\mathrm{C}_{3} \mathrm{H}_{7}$

$\underline{\mathbf{R}}^{\mathbf{1}}$

morpholino $\quad$ ii
morpholino
piperidino
pyrrolidino
pyrrolidino

4a-e<smiles>[X]C(C#N)=Cc1c([R])nn(CCCCCC)c1[R]</smiles>

5a-e

$\underline{\mathbf{R}}$

$\underline{R}^{1}$

$\underline{\mathbf{X}}$

a $\mathrm{CH}_{3}$ morpholino $\mathrm{CN}$

b $\mathrm{C}_{6} \mathrm{H}_{5}$ morpholino $\mathrm{CN}$

c $\mathrm{C}_{6} \mathrm{H}_{5}$ piperidino $\mathrm{CN}$

d $\mathrm{C}_{2} \mathrm{H}_{5}$ pyrrolidino $\mathrm{CONH}_{2}$

e $\mathrm{C}_{3} \mathrm{H}_{7}$ pyrrolidino $\mathrm{CSNH}_{2}$

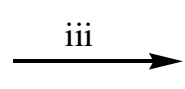<smiles>[R]c1nn(C)c2c1CC([X])(C)C1C[Y]CN21</smiles>

6a-e

\begin{tabular}{llll} 
& $\underline{\mathbf{R}}$ & \multicolumn{1}{r}{} & $\underline{\mathbf{x}}$ \\
a & $\mathrm{CH}_{3}$ & $\mathrm{CH}_{2} \mathrm{O}$ & $\mathrm{CN}$ \\
b & $\mathrm{C}_{6} \mathrm{H}_{5}$ & $\mathrm{CH}_{2} \mathrm{O}$ & $\mathrm{CN}$ \\
c & $\mathrm{C}_{6} \mathrm{H}_{5}$ & $\mathrm{CH}_{2} \mathrm{CH}_{2}$ & $\mathrm{CN}$ \\
d & $\mathrm{C}_{2} \mathrm{H}_{5}$ & $\mathrm{CH}_{2}$ & $\mathrm{CONH}_{2}$ \\
e & $\mathrm{C}_{3} \mathrm{H}_{7}$ & $\mathrm{CH}_{2}$ & $\mathrm{CSNH}_{2}$
\end{tabular}

Scheme-I: (i) cyclic sec-amine, dry EtOH, dry $\mathrm{Et}_{3} \mathrm{~N}$, reflux, 17-22 h; (ii) $\mathrm{CNCH}_{2} \mathrm{X}$, dry EtOH, reflux, 5-8 h; (iii) $\mathrm{ZnCl}_{2}$, dry toluene, 7-17 h

prepared from the pyrazolones employing Vilsmeier-Haack chloroformylation ${ }^{24}$ by heating pyrazolones with an excess phosphorus oxychloride in DMF. The structures of compounds 3a-d were confirmed from their IR, ${ }^{1} \mathrm{H}$ NMR, ${ }^{13} \mathrm{C}$ NMR and MS spectra data. Compounds 3a-d were subjected to aromatic nucleophilic substitution with several cyclic sec-amines (viz. pyrrolidine, piperidine and morpholine) resulted in smooth conversion to the 3,5-disubstituted 1-phenyl-1 $H$-pyrazole-4carbaldehyde $\mathbf{4 a -}-\mathbf{e}^{25}$. The structures of $\mathbf{4 a - e}$ were assigned on the basis of spectroscopic analyses. Thus, IR spectrum showed band at $1669-1628 \mathrm{~cm}^{-1}$ for $\mathrm{C}=\mathrm{O}$ stretching, in addition to bands in the range $2878-2738 \mathrm{~cm}^{-1}$ which are characteristic to $\mathrm{C}-\mathrm{H}$ stretching of aldehyde group. Mass spectral data have been found to be in conformity with the assigned structure. The ${ }^{1} \mathrm{H}$ NMR spectrum of $\mathbf{4 a}$ showed two triplets $(J=4.7)$, each integrated for 4 protons at $\delta 3.13 \mathrm{ppm}\left(\mathrm{CH}_{2}-\mathrm{N}_{-} \mathrm{CH}_{2}\right)$ and $3.67 \mathrm{ppm}\left(\mathrm{CH}_{2}-\mathrm{O}-\mathrm{CH}_{2}\right)$ beside the one proton singlet at $\delta 9.95$ ppm for the CHO. The chemical shifts of other proton absorptions in the latter spectrum of $\mathbf{4 a}$ as well as the whole carbon signals in the ${ }^{13} \mathrm{C}$ NMR spectrum were in complete consistent with its structure.

Compounds 4a-e were then used in the Knoevenagel condensation reactions with compounds containing active methylene group, namely, malononitrile, cyanoacetamide and cyanothioacetamide to give the corresponding olefinic products 5a-e, where intramolecular cyclization occurs between the $\beta$-carbon of a vinylic group possessing electron withdrawing substituents at the $\beta$-position and $\alpha$-carbon of an tert-amino group in the presence of anhydrous zinc chloride to get the corresponding pyrazolopyridooxazine, pyrazoloquinolizines and pyrazoloindolizine derivatives $\mathbf{6 a}-\mathbf{e}^{25}$. The structures of 5a-e were confirmed by the analysis of its various spectroscopic data. Its IR spectrum showed an band at 2225-2204 $\mathrm{cm}^{-1}$ attributed for $\mathrm{CN}$ stretching and band at $1645-1578 \mathrm{~cm}^{-1}$ due to $\mathrm{C}=\mathrm{C}$ stretching. Mass spectral data have been found to be in conformity with the assigned structure. The ${ }^{1} \mathrm{H}$ NMR spectra of 5a-e revealed singlet at range $\delta$ 7.66-8.95 ppm for the olefinic proton. The spectrum of $\mathbf{5 d}$ also showed two broad singlet at $\delta 5.93$ and $6.25 \mathrm{ppm}$ for the $\mathrm{NH}_{2}$, because the two protons are in magnetically different environments and have slightly different chemical shifts ${ }^{28}$. All other proton signals in the latter compounds were in consistence with their structures (Experimental Section). The ${ }^{13} \mathrm{C}$ NMR spectra of these compounds displayed signals for all carbons atoms in the molecule. The spectra showed two signals at $\delta$ 77.1-101.2 ppm and $\delta$ 146.1-151.8 ppm assigned to two carbons at positions 2 and 3 , respectively, while $\mathrm{CN}$ appeared in the range $\delta 112.1-117.3$ ppm. 
The structures of 6a-e were characterized by high resolution spectral analysis of DEPT, ${ }^{1} \mathrm{H}-{ }^{1} \mathrm{H}$ Cosy and ${ }^{1} \mathrm{H}-{ }^{13} \mathrm{C}$ Cosy techniques. Their IR spectra showed bands at 2229-2253 $\mathrm{cm}^{-1}$ attributed for $\mathrm{CN}$ stretching. The ${ }^{1} \mathrm{H}$ NMR spectra of 6a-e showed the absence of the olefinic protons. The IR, ${ }^{1} \mathrm{H}$ NMR, ${ }^{13} \mathrm{C}$ NMR and MS spectral data of 6a-e were in complete agreement with their structures.

Treatment of 2e-f with 2-arylidenemalononitriles 8a-c (Scheme-II), either by stirring overnight ${ }^{27}$ or one pot four components synthesis using ultrasound irradiation in aqueous medium in the presence of catalyst and using microwave irradiation yielded the same product 9a-c (IR, TLC, m.p. and mixed m.p.). IR spectra of 9a-c displayed characteristic bands at 2189-2209, 3314-3355 and 3380-3466 $\mathrm{cm}^{-1}$, due to absorption of $\mathrm{CN}$ and $\mathrm{NH}_{2}$ groups, respectively. The mass spectra of these compounds were in accordance with the assigned structures. The ${ }^{1} \mathrm{H}$ NMR spectrum of 9 a showed a singlet at $\delta$ 4.66 for $\mathrm{H}-4$, broad singlet at $\delta 7.08 \mathrm{ppm}\left(\mathrm{D}_{2} \mathrm{O}\right.$ exchangeable) for $\mathrm{NH}_{2}$ besides the other proton signals in the latter compounds which were in consistence with its structure (experimental section). The assignment of all protons and carbons in 9a were verified by the aid of the analysis of DEPT, ${ }^{1} \mathrm{H}-{ }^{1} \mathrm{H}$ Cosy and ${ }^{1} \mathrm{H}-{ }^{13} \mathrm{C}$ Cosy techniques.

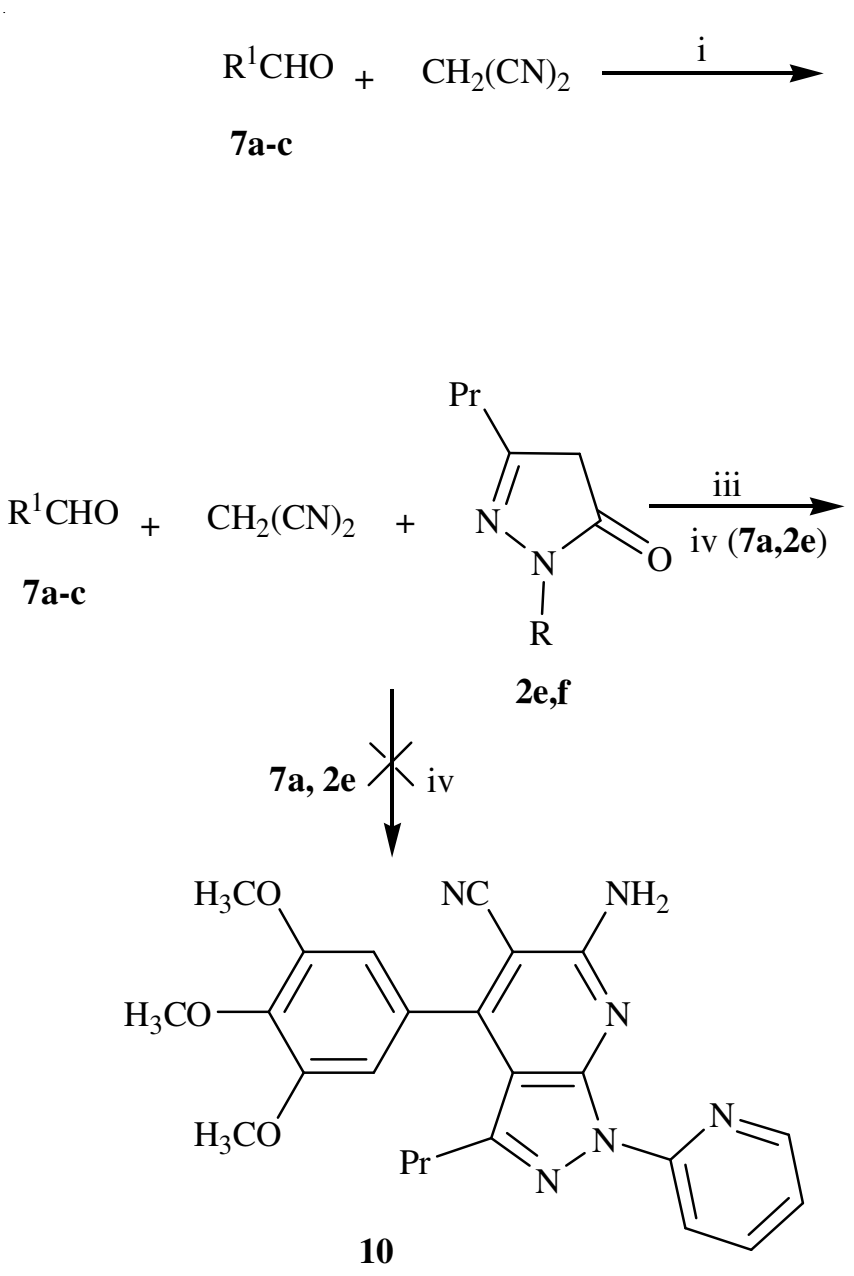

Attempts were made to prepare 6-amino-3-propyl-1-(2pyridinyl)-4-(3',4',5'-trimethoxy-phenyl)-1H-pyrazolo[3,4b]pyridine-5-carbonitrile (10) by one pot four component reaction using traditional reflux method, microwave and ultrasound irradiation of mixture aldehyde $\mathbf{7 a}$, pyrazolone $\mathbf{2 e}$ and malononitrile in the presence of ammonium acetate were not successful ${ }^{26}$. Instead we obtained the same product 9a as indicated by the ${ }^{1} \mathrm{H}$ NMR data of the reaction product (SchemeII).

Cyclization of 9a,b with acetic anhydride in the presence of conc. $\mathrm{H}_{2} \mathrm{SO}_{4}$ afforded pyrano[2,3-d]pyrimidin-5-one derivatives $\mathbf{1 1 a}, \mathbf{b}^{26}$ (Scheme-II). IR spectrum of these compounds exhibited the absence of stretching band of cyano group and a broad absorption bands of the $\mathrm{NH} / \mathrm{OH}$ stretching in the region of 3484-3449 $\mathrm{cm}^{-1}$ beside bands at the range of 1625-1655 $\mathrm{cm}^{-1}$ due to $\mathrm{C}=\mathrm{O}$ stretching. All other spectral analysis match the structures of the prepared 11a,b.

\section{Conclusion}

We have synthesized a series of novel pyrazolopyridooxazine, pyrazoloquinolizines and pyrazoloindolizine derivatives from the intermolecular $\alpha$-cyclization of tertiary amines in good yields. We have also demonstrated the use one pot four compo-

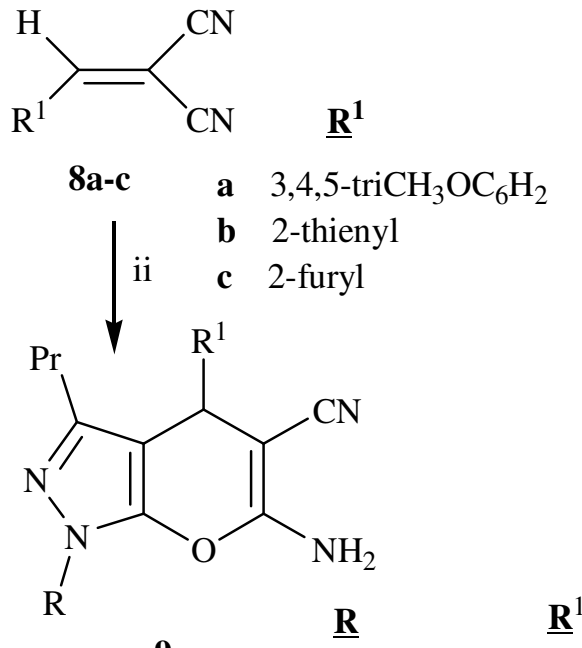<smiles>[R7]c1nn([R])c2c1C([R])c1c(nc(C)[nH]c1=O)O2</smiles>

$\begin{array}{ccc}\text { 11a,b } & \underline{\mathbf{R}} & \underline{\mathbf{R}}^{\mathbf{1}} \\ \text { a } & \text { 2-pyridinyl } & \text { 3,4,5-triCH}{ }_{3} \mathrm{OC}_{6} \mathrm{H}_{2} \\ \text { b } & \mathrm{CH}_{3} & \text { 2-thienyl }\end{array}$

Scheme-II: (i) $\mathbf{1}$ drop piperidine, EtOH, reflux, $15 \mathrm{~min}$; (ii) $\mathbf{2 e , f}, \mathrm{MeOH}$, morpholine, overnight; (iii) a: piperidine, $\mathrm{MW}, 5-30 \mathrm{~min}$; b: CTACl, $\mathrm{H}_{2} \mathrm{O}$, US, 1.5 h; (iv) $\mathrm{NH}_{4} \mathrm{OAc}$, a: EtOH, reflux 5 h; b: MW, 3 min; c: EtOH, US, 1 h; (v) $\mathrm{Ac}_{2} \mathrm{O}$, heat, $\mathrm{H}_{2} \mathrm{SO}_{4}$, room temperature, $24 \mathrm{~h}$ 
nents reaction using traditional reflux, microwave and ultrasound irradiation methods for the synthesis the pyrano[2,3c]pyrazole derivatives. Cyclization of the later derivatives afforded pyrazolopyranopyrimidinone derivatives.

\section{ACKNOWLEDGEMENTS}

This research project was supported by a grant from the Research Center of the Center for Female Scientific and Medical Colleges in King Saud University.

\section{REFERENCES}

1. I. Çelik, N. KanIskan and S. Kökten, Tetrahedron, 65, 328 (2009).

2. T.D. Penning, J.J. Talley, S.R. Bertenshaw, J.S. Carter, P.W. Collins, S. Docter, M.J. Graneto, L.F. Lee, J.W. Malecha, J.M. Miyashiro, R.S. Rogers, D.J. Rogier, S.S. Yu, G.D. Anderson, E.G. Burton, J.N. Cogburn, S.A. Gregory, C.M. Koboldt, W.E. Perkins, K. Seibert, A.W. Veenhuizen, Y.Y. Zhang and P.C. Isakson, J. Med. Chem., 40, 1347 (1997).

3. M. Rinaldi-Carmona, F. Barth, M. Héaulme, D. Shire, B. Calandra, C. Congy, S. Martinez, J. Maruani, G. Néliat, D. Caput, P. Ferrara, P. Soubrié, J.C. Brelière and G. Le Fur, FEBS Lett., 350, 240 (1994).

4. H.A. DeWald, S. Lobbestael and B.P.H. Poschel, J. Med. Chem., 24, 982 (1981).

5. K.S. Girisha, B. Kalluraya and V. Narayana, Eur. J. Med. Chem., 45, 4640 (2010)

6. A.M. Isloor, B. Kalluraya and K. Sridhar Pai, Eur. J. Med. Chem., 45, 825 (2010).

7. A.M. Youssef, M. Sydney White, E.B. Villanueva, I.M. El-Ashmawy and A. Klegeris, Bioorg. Med. Chem., 18, 2019 (2010).

8. P.-C. Lv, J. Sun, Y. Luo, Y. Yang and H.-L. Zhu, Bioorg. Med. Chem. Lett., 20, 4657 (2010).

9. S. Bondock, R. Rabie, H.A. Etman and A.A. Fadda, Eur. J. Med. Chem., 43, 2122 (2008)

10. X.-H. Liu, J. Zhu, A. Zhou, B.-A. Song, H.-L. Zhu, L.-S. Bai, P.S Bhadury and C.-X. Pan, Bioorg. Med. Chem., 17, 1207 (2009).
11. N.P. Rai, V.K. Narayanaswamy, S. Shashikanth and P.N. Arunachalam, Eur. J. Med. Chem., 44, 4522 (2009).

12. H.A. Abdel-Aziz, A.A.I. Mekawey and K.M. Dawood, Eur. J. Med. Chem., 44, 3637 (2009).

13. A.E. Rashad, M.I. Hegab, R.E. Abdel-Megeid, N. Fathalla and F.M.E. Abdel-Megeid, Eur. J. Med. Chem., 44, 3285 (2009).

14. A.E. Rashad, M.I. Hegab, R.E. Abdel-Megeid, J.A. Micky and F.M.E. Abdel-Megeid, Bioorg. Med. Chem., 16, 7102 (2008).

15. M.M. Ghorab, F.A. Ragab, S.I. Alqasoumi, A.M. Alafeefy and S.A. Aboulmagd, Eur. J. Med. Chem., 45, 171 (2010).

16. H.A. Abdel-Aziz, H.S.A. El-Zahabi and K.M. Dawood, Eur. J. Med. Chem., 45, 2427 (2010).

17. B.P. Bandgar, J.V. Totre, S.S. Gawande, C.N. Khobragade, S.C. Warangkar and P.D. Kadam, Bioorg. Med. Chem., 18, 6149 (2010).

18. F. El-Baih and H.M. Al-Hazimi, Asian J. Chem., 23, 1309 (2011).

19. A.M. Farag, K.A.K. Ali, T.M.A. El-Debss, A.S. Mayhoub, A.-G.E. Amr, N.A. Abdel-Hafez and M.M. Abdulla, Eur. J. Med. Chem., 45, 5887 (2010).

20. S.K. Meegalla, D. Doller, G.M. Silver, N. Wisnewski, R.M. Soll and D. Dhanoa, Bioorg. Med. Chem. Lett., 13, 4035 (2003).

21. G.P. Lahm, T.M. Stevenson, T.P. Selby, J.H. Freudenberger, D. Cordova, L. Flexner, C.A. Bellin, C.M. Dubas, B.K. Smith, K.A. Hughes, J.G. Hollingshaus, C.E. Clark and E.A. Benner, Bioorg. Med. Chem. Lett., 17, 6274 (2007).

22. S. Gupta, A. Sivasubramanian, L.M. Rodrigues, A.P. Esteves, R. Hrdina and A.M.F. Oliveira-Campos, Dyes Pigments, 75, 82 (2007).

23. A.A. Al-Mutairi, F.E.M. El-Baih and H.M. Al-Hazimi, J. Saudi Chem. Soc., 14, 287 (2010).

24. J. Becher, P.L. Joergensen, K. Pluta, N.J. Krake and B. Falt-Hansen, J. Org. Chem., 57, 2127 (1992).

25. D. Prajapati and K.J. Borah, Beilstein J. Org. Chem., 3, 43 (2007)

26. T.M. Al-Mutairi, H.M. Al-Hazimi and F.E.M. El-Baih, J. Saudi Chem. Soc., 13, 199 (2009).

27. Y.A. Sharanin, V.K. Promonenkov and L.G. Sharanina, J. Org. Chem. (USSR), 18, 544 (1982).

28. D.L. Pavia, G.M. Lampman, G.S. Kriz and J.R. Vyvyan, Introduction to Spectroscopy, Thmoson Learning, edn. 4 (2009). 